\title{
Bioinformatics analysis of microarray data to reveal the pathogenesis of diffuse intrinsic pontine glioma
}

\author{
Li Wei $^{1+}$, Fei He ${ }^{1+}$, Wen Zhang ${ }^{1}$, Wenhua Chen ${ }^{1,2}$ and Bo Yu ${ }^{1,2^{*}}$
}

\begin{abstract}
Background: Diffuse intrinsic pontine glioma (DIPG) is the main cause of pediatric brain tumor death. This study was designed to identify key genes associated with DIPG.

Methods: The gene expression profile GSE50021, which consisted of 35 pediatric DIPG samples and 10 normal brain samples, was downloaded from the Gene Expression Omnibus database. Differentially expressed genes (DEGs) were identified by limma package. Functional and pathway enrichment analyses were performed by the DAVID tool. Protein-protein interaction (PPI) network, and transcription factor (TF)-microRNA (miRNA)-target gene network were constructed using Cytoscape. Moreover, the expression levels of several genes were validated in human glioma cell line U251 and normal glia HEB cells through real-time polymerase chain reaction (PCR).

Results: A total of 378 DEGs were screened (74 up-regulated and 304 down-regulated genes). In the PPI network, GRM1, HTR2A, GRM7 and GRM2 had higher degrees. Besides, GRM1 and HTR2A were significantly enriched in the neuroactive ligand-receptor interaction pathway, and calcium signaling pathway. In addition, TFAP2C was a significant down-regulated functional gene and $h s a-m i R-26 b-5 p$ had a higher degree in the TF-miRNA-target gene network. PCR analysis revealed that GRM7 and HTR2A were significantly downregulated while TFAP2C was upregulated in U251 cells compared with that in HEB cells $(p<0.001)$. GRM2 was not detected in cells.
\end{abstract}

Conclusions: GRM1 and HTR2A might function in DIPG through the neuroactive ligand-receptor interaction pathway and the calcium signaling pathway. Furthermore, the TFAP2C and $h s a-m i R-26 b-5 p$ might play important roles in the development and progression mechanisms of DIPG.

Keywords: Diffuse intrinsic pontine glioma, Differentially-expressed genes, Neuroactive ligand-receptor interaction, Protein-protein interaction network, Transcription factor-microRNA-target gene network

\section{Background}

Diffuse intrinsic pontine glioma (DIPG) is the most common brain tumor in childhood [1]. The mortality of DIPG goes up with no available treatment, almost $100 \%$ fatality [2]. Although DIPG can be treated by radiotherapy and chemotherapy, the average survival time has remained only 9 months and 5 -year survival time is less than $1 \%$ [3]. There were no obviously advantages of radiation

\footnotetext{
*Correspondence: Bobby_JTU@163.com

${ }^{\dagger}$ Li Wei and Fei He should be regard as co-first authors

${ }^{1}$ Department of Rehabilitation, Shanghai General Hospital, Shanghai Jiao

Tong University, No. 100, Haining Road, Shanghai 200080, China

Full list of author information is available at the end of the article
}

and chemotherapy [4]. The development of therapies for DIPG was greatly hampered because of lack of therapeutic benefits and molecular studies [5]. Therefore, a better understanding of the molecular mechanisms underlying DIPG is helpful to develop new therapies for this disease.

In the past few years, DIPG cell cultures and orthotopic xenograft models have been established [6, 7]. Previous study showed that grade of gliomas in human brain was related to the $R$-Ras expression and phosphorylation, indicating the EphB2/R-Ras signaling pathway as a potential target associated with cell adsorption, growth and invasion [8]. Holland et al. [9] found that activation of Ras and $A k t$ in neural progenitor cells can induce 
glioma in mice, and the Ras and Akt proteins play important roles in the pathogenesis of gliomas. These studies suggest that a single gene or the interaction between more genes involved in the promotion of disease occurrence and development. In recent years, the advantage of gene chip technology and bioinformatics analysis is obviously observed, which is applied to analyze the molecular mechanism of DIPG [10]. Deng et al. [10] showed cholecystokinin (CCK) and gastrin (GAST) associated with the G-protein coupled receptor (GPCR) signaling pathway, and 5-hydroxytryptamine (serotonin) receptor 7 (HTR7) involved in the neuroactive ligand-receptor interaction might play critical roles in DIPG. Despite a number of researches have investigated the molecular basis of DIPG, the molecular mechanisms of the disease remain not fully understood.

In the study of Buczkowicz et al. [2], the gene expression profile GSE50021 was utilized only for surveying what urged DIPGs by whole-genome sequencing. In the study of Deng et al. [10], GSE50021 was analyzed and revealed a potential key molecular mechanisms in DIPG by microarray analysis and bioinformatics analysis. Recently, Xi et al. [11] used a novel method for extracting DEGs from GSE50021 in combination with GSE50022 that included DNA methylation. However, given the complicated molecular mechanisms of DIPG, it is necessary to fully utilize GSE50021 profile to identify more potential genes and pathways related to DIPG. In this study, differentially-expressed genes (DEGs) were indentified from GSE50021 dataset. Subsequently, enrichment analysis, protein-protein interaction (PPI) network, module analyses, and microRNAs (miRNAs)-transcription factors (TFs)-target gene regulatory network analysis were successively performed to identify the key genes implicated in the pathogenesis of DIPG. Importantly, several key genes were validated through real-time polymerase chain reaction (PCR).

\section{Methods}

\section{Microarray data}

The gene expression profile GSE50021 was downloaded from the Gene Expression Omnibus (http://www.ncbi. nlm.nih.gov/geo/) database [12], which was based on the platform of GPL13938 Illumina HumanHT-12 WGDASL V4.0 expression beadchip. This dataset, including 35 DIPG samples and 10 normal brain samples, was deposited by Buczkowicz et al. [2].

\section{Data preprocessing and DEGs screening}

Using robust multi-array average (RAM) [13] method of Affy package (http://www.bioconductor.org/packages/ release/bioc/html/affy.html) in $\mathrm{R}$ language, the raw data were preprocessed, including background correction, normalization and expression calculation. The platform annotation file was used to annotate the probes, and the probes without corresponding gene symbols were removed. For different probes mapped to the same gene, the average value of the probes was taken as the final gene expression value. DEGs were identified by the classical Bayes method in Limma package [14] (http://www. bioconductor.org/packages/2.9/bioc/html/limma.html). The genes with adjusted $p$-value $<0.05$ were chosen as DEGs.

\section{Functional and pathway enrichment analyses}

Gene Ontology (GO) (http://www.geneontology.org) analysis [15], including biological process (BP), molecular function (MF), and cellular component (CC), is used for functional study of single gene or large-scale genome. The Kyoto Encyclopedia of Genes and Genomes (KEGG, http://www.genome.ad.jp/kegg/) [16, 17] is the major recognized pathway-related database, which takes into account not only each KEGG pathway itself, but also its related pathways [17]. The DAVID online tool (https ://david-d.ncifcrf.gov/) [18] was used to perform GO functional and KEGG pathway enrichment analysis for the DEGs. The $p$-value of $<0.05$ and gene count $\geq 2$ were chosen as the significant thresholds. In order to directly observe the functions of DEGs, the ClueGO plug-in $[19,20]$ http://apps.cytoscape.org/apps/ClueGO) of Cytoscape [21] was applied to visualize the results of enrichment analysis in figures, and $p$-value of $<0.05$ was chosen as the significant threshold.

\section{PPI network and module analyses}

The Search Tool for the Retrieval of Interacting Genes (STRING) [22] (http://www.string-db.org/) is an online database providing experimental and predicted PPI information. In this study, the STRING database [22] was used to analyze the PPIs among the proteins encoded by the DEGs with a combined score of $>0.4$, then the PPI networks for the up-regulated and the down-regulated genes were separately visualized by Cytoscape software (http:// www.cytoscape.org/) [21]. The CytoNCA plug-in [23] (http://apps.cytoscape.org/apps/cytonca) in Cytoscape software was used to analyze the topological property of the network, acquiring the important nodes in the PPI network combined with the degree of each node.

In addition, module analysis was performed for the PPI networks using the MCODE plug-in (http://apps.cytos cape.org/apps/mcode) [24] in Cytoscape software. An adjusted $\mathrm{p}<0.01$ was chosen as the significance threshold. In addition, the nodes in the significant modules were performed GO functional and KEGG pathway enrichment analyses using DAVID online tool [18]. 
Construction of TF-miRNA-target gene regulatory network At the post-transcription stage, miRNAs regulate gene expression [25]. Whereas, TFs can promote or repress transcription at a pre-transcription stage [26]. TFmiRNA-target gene acts as a tumor suppressor network, triggering a comprehensive change in genetic programs involving cell proliferation, apoptosis and cancer invasion in cancer [27]. The miRNAs associated with DIPG and their target genes were searched using miRWalk2.0 database [28] (http://zmf.umm.uni-heide lberg.de/apps/zmf/mirwalk2/). Through comparing target genes with the DEGs, miRNA-DEG pairs were obtained. Then, miRNA-DEG regulatory network was visualized by Cytoscape software [21].

The iRegulon plug-in [29] (http://apps.cytoscape.org/ apps/iRegulon) in Cytoscape software, which included the TF-target pairs of multiple human databases such as Transfac (http://www.gene-regulation.com/pub/ databases.html) [30], and Encode (https://www.encod eproject.org/) [31, 32], was used to predict the TFtarget pairs in the miRNA-DEG regulatory network, A normalized enrichment score $(\mathrm{NES})>3$ was chosen as the significant threshold for screening TF-target pairs. Additionally, the TF-miRNA-target regulatory network was visualized using Cytoscape software [21].

\section{Real-time PCR verification of the expression of key genes} Human glioma cell line U251, a common used DIPG cell line [33], was purchased from cell bank of Chinese Academy of Sciences (Shanghai, China) and normal glia HEB cells [33] were purchased from GuangZhou Jennio Biotech Co., Ltd, Guangdong, China. All cells were grown in Dulbecco's modified eagle medium supplemented with $10 \%$ fetal bovine serum and $1 \%$ antibiotics (penicillin and streptomycin) at $37{ }^{\circ} \mathrm{C}$ in an atmosphere of $10 \% \mathrm{CO}_{2}$.

Briefly, total RNAs were isolated from $5 \times 10^{6}$ to $10 \times 10^{6}$ cell samples using a TRIzol reagent (Invitrogen, CA, USA). RNA concentration and quality were determined using a TECAN infinite M100 PRO Biotek microplate reader (TECAN, CA, USA). Total RNA $(0.5 \mu \mathrm{g})$ was used for cDNA synthesis using the PrimeScript RT Master Mix (RR036A; Takara, Dalian, China). PCR was performed using the SYBR GREEN kit (4367659; Thermo, USA) in Viia7 Real-Time PCR System (Applied Biosystems, USA). The primers used in this study are listed in Table 1.

\section{Statistical analysis}

Data are presented as mean \pm standard deviation. Statistical analysis was performed using SPSS 22.0 (IBM, Armonk, NY, USA). Differences in gene expression
Table 1 The primers used in real time PCR

\begin{tabular}{ll}
\hline Primer name & Sequences $\left(\mathbf{5}^{\prime} \mathbf{- 3}^{\prime} \mathbf{)}\right.$ \\
\hline GRM2-hF & GCTCCACTCCGATTCTCTCC \\
GRM2-hR & GAAGCAGCGAAGGCAAAGAG \\
GRM7-hF & GACACTTACGCGCTCGAACA \\
GRM7-hR & TCATCACTTAGCTCGGGTGC \\
HTR2A-hF & CTGGTCTGCTTACTGACAGCC \\
HTR2A-hR & AGAGCACGTCCAGGTAAATCC \\
GAPDH-hF & TGACAACTTTGGTATCGTGGAAGG \\
GAPDH-hR & AGGCAGGGATGATGTTCTGGAGAG \\
\hline
\end{tabular}

levels between groups were analyzed by one-way analysis of variance. The $\mathrm{p}<0.05$ was considered significant.

\section{Results \\ DEGs screening}

As shown in Fig. 1, the medians located at the same level after performing data normalization, which indicated a perfect effect. Based on adjusted $p$-value $<0.05,378$ DEGs were identified, which included 74 up-regulated and 304 down-regulated genes.

\section{Functional and pathway enrichment analyses}

According to the $p$-values (ascending sort), the top five enriched terms are exhibited in Fig. 2. The up-regulated genes were significantly enriched in the modification-dependent macromolecule catabolic process (BP, $p=0.044$ ), nucleotide binding (MF, $p=0.007$ ), cytosolic part $(\mathrm{CC}, p=0.044)$, and antigen processing and presentation (pathway, $p=0.01$ ) (Fig. 2a). While the down-regulated genes were significantly associated with neurological system process (BP, $p=2.33 \mathrm{E}-08$ ), ion channel activity (MF, $p=6.64 \mathrm{E}-09$ ), plasma membrane part (CC, $p=1.48 \mathrm{E}-08)$, neuroactive ligand-receptor interaction (pathway, $p=1.57 \mathrm{E}-08$ ) and calcium signaling pathway (pathway, $p=4.18 \mathrm{E}-06$ ) (Fig. $2 \mathrm{~b}$ ).

Furthermore, the crosslinking enrichment of GO-BP terms and KEGG pathways are shown in Fig. 3. The more down-regulated genes were related to the disease, and the main pathway was neuroactive ligand-receptor interaction (Fig. 3).

\section{PPI network and module analyses}

Based on the STRING database, the PPI network for the DEGs (including 231 nodes and 490 edges) was constructed (Fig. 4). Up-regulated gene with higher node degree was glyceraldehyde-3-phosphate dehydrogenase $(G A P D H)$. Down-regulated genes had higher degrees were as follows: nerve peptide Y $(N P Y)$, 


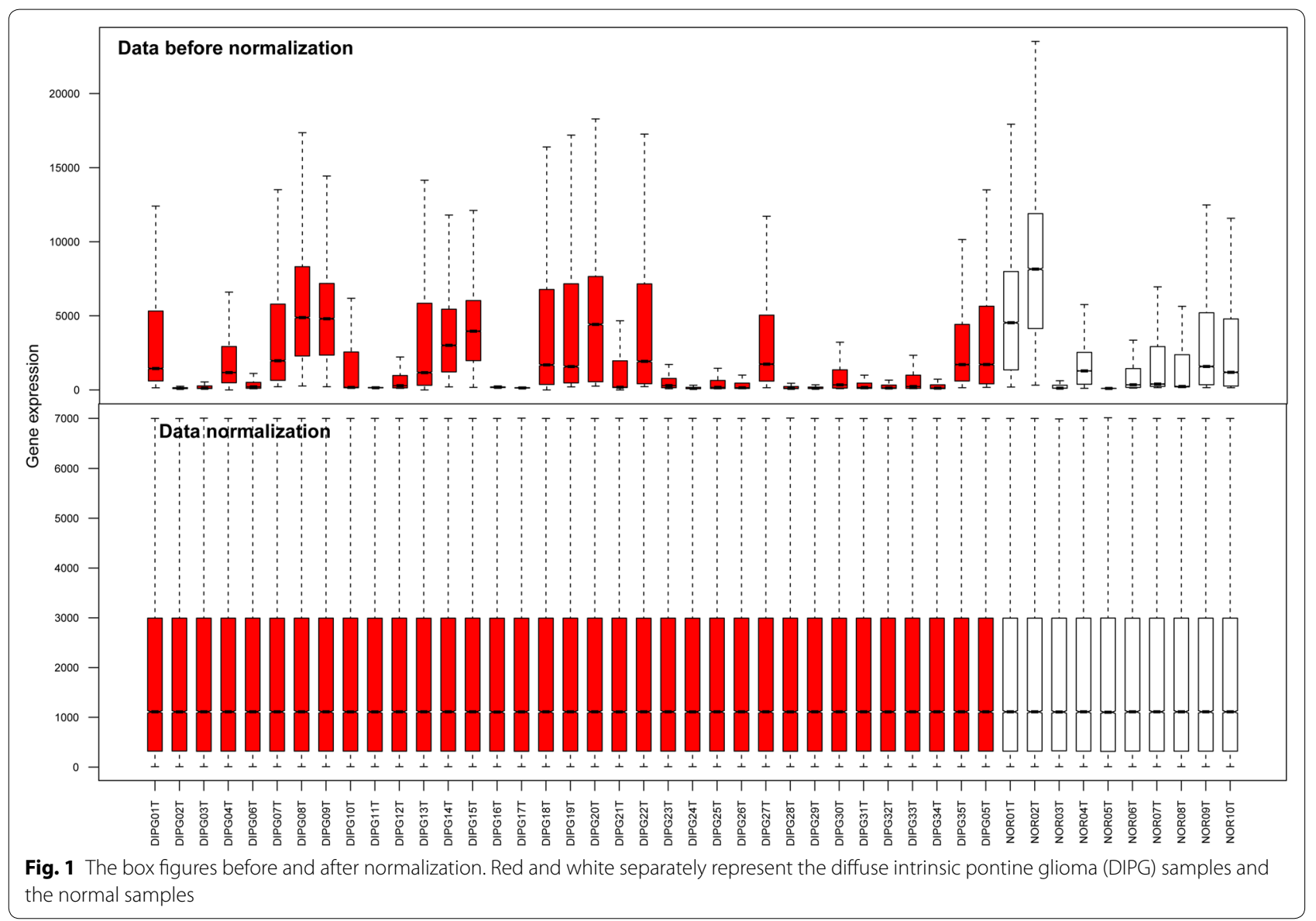

5-hydroxytryptamine receptor 2A (HTR2A), metabotropic glutamate receptor 1 (GRM1), adenylate cyclase 2 (ADCY2), GRM2, GRM7 and so on. The nodes with degree $\geq 10$ are listed in Table 2 .

Additionally, three significant modules, including module 1 (19 nodes and 95 edges), module 2 (5 nodes and 10 edges) and module 3 (9 nodes and 15 edges), were acquired by MCODE plug-in (Fig. 4).

Furthermore, 10 KEGG pathways were significantly enriched by module 1 , including neuroactive ligandreceptor interaction $(p=7.94 \mathrm{E}-12)$, calcium signaling $(p=1.83 \mathrm{E}-05)$, glutamatergic synapse $(p=1.39 \mathrm{E}-03)$. Meanwhile, the nodes in module 3 were significantly enriched in four KEGG pathways, including cocaine addiction $(p=2.37 \mathrm{E}-06)$, nicotine addiction $(p=6.74 \mathrm{E}-04)$, cAMP signaling pathway $(p=1.56 \mathrm{E}-02)$ and glutamatergic synapse $(p=4.86 \mathrm{E}-02)$ (Table 3$)$. However, no pathways were enriched for the nodes in module 2 .

\section{TF-miRNA-target regulatory network analysis}

A total of 36 miRNAs associated with DIPG were identified from miRWalk2.0, and only 27 miRNAs remained after removing the repeats and the miRNAs in mice. The target genes of the remaining miRNAs were compared with the DEGs and a total of 141 miRNA-DEG pairs were obtained. The miRNA-DEG regulatory network was visualized by Cytoscape software, consisting of 136 nodes and 368 edges (Fig. 5). Based on the iRegulon plug-in, a total of nine TFs were identified from the miRNA-DEG regulatory network. Then, the TFmiRNA-target regulatory network was constructed and the nodes with top 10 degrees are listed in Table 4. The TFAP2C was a significant down-regulated functional gene, while the $h s a-m i R-26 b-5 p$ had a higher degree in the TF-miRNA-target regulatory network.

\section{Real-time PCR verification of the expression of key genes}

Expression levels of GRM2, GRM7, HTR2A and TFAP2C were determined using real-time PCR. As shown in Fig. 6, GRM7 and HTR2A were significantly downregulated while TFAP $2 C$ was significantly upregulated in U251 cells compared with that in HEB cells $(\mathrm{p}<0.001)$. GRM2 was not detected in cells, which may 
a

b
GO:0016879 ligase activity, forming carbon-nitrogen bonds GO:0016881 acid-amino acid ligase activity GO:0000166 nucleotide binding GO:0019787 small conjugating protein ligase activity GO:0004842 ubiquitin-protein ligase activity GO:0022626 cytosolic ribosome GO:0015934 large ribosomal subunit GO:0044445 cytosolic part GO:0022625 cytosolic large ribosomal subunit GO:0032269 negative regulation of cellular protein metabolic process GO:0019941 modification-dependent protein catabolic process GO:0043632 modification-dependent macromolecule catabolic process GO:0006414 translational elongation hsa04120:Ubiquitin mediated proteolysis hsa04612:Antigen processing and presentation

$$
0
$$

GO:0022836 gated channel activity GO:0022803 passive transmembrane transporter activity GO:0015267 channel activity GO:0022838 substrate specific channel activity GO:0005216 ion channel activity GO:0043005 neuron projection GO:0045211 postsynaptic membrane GO:0044459 plasma membrane part GO:0044456 synapse part GO:0045202 synapse GO:0050877 neurological system process GO:0006811 ion transport GO:0007267 cell-cell signaling GO:0019226 transmission of nerve impulse GO:0007268 synaptic transmission hsa05410:Hypertrophic cardiomyopathy (HCM) hsa 04720:Long-term potentiation hsa05414:Dilated cardiomyopathy hsa04270:Vascular smooth muscle contraction hsa 04020:Calcium signaling pathway hsa04080:Neuroactive ligand-receptor interaction

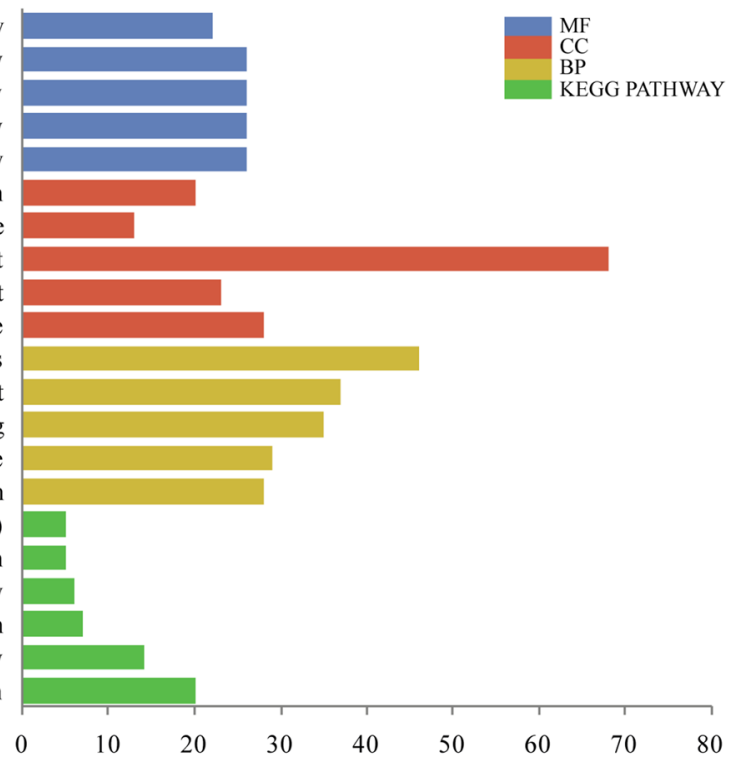

Fig. 2 The top five GO terms and KEGG pathways enriched separately for the up-regulated genes (a) and the down-regulated (b). MF molecular function, BP biological processes, CC cellular components, KEGG Kyoto Encyclopedia of Genes and Genomes, GO gene ontology; The horizontal axis represents the count of enriched DEGs. The vertical axis represents the enriched GO terms and KEGG pathways

be due to its expression level being too low in them or the difference between tissue and cell samples.

\section{Discussion}

In order to identify potential genes and related pathways of DIPG, a further analysis based on bioinformatics method was performed on the GSE50021 profile. Deng et al. [10] also used GSE50021 profile to analyze candidate genes and associated pathways, and identified 679 DEGs (454 up-regulated and 225 down-regulated genes) but the results had both similarities and dissimilarities on the number and function of DEGs. In the present study, 378 DEGs were identified, including 74 up-regulated genes and 304 down-regulated genes. The difference on the number of DEGs is due to the different thresholds of DEGs screening. In the study of Deng et al. [10], genes with $p$-value of $<0.01$ and $\mid \log$ 2 fold change (FC) $\mid>2.0$ were selected as DEGs for further study, while our study used adjusted $p$-value $<0.05$ as threshold. Based on the module analysis, the downregulated genes were significantly enriched in different GO terms and pathways, for instance, the GRM1 and $H T R 2 A$ were associated with neuroactive ligandreceptor interaction and calcium signaling pathway, while the $A D C Y 2$ was associated with calcium signaling pathway, in addition, $N P Y$ with higher degree in PPI network. TF-miRNA-target gene network showed that TFAP2C was a significantly down-regulated functional gene and the $h s a-m i R-26 b-5 p$ had a higher degree. 


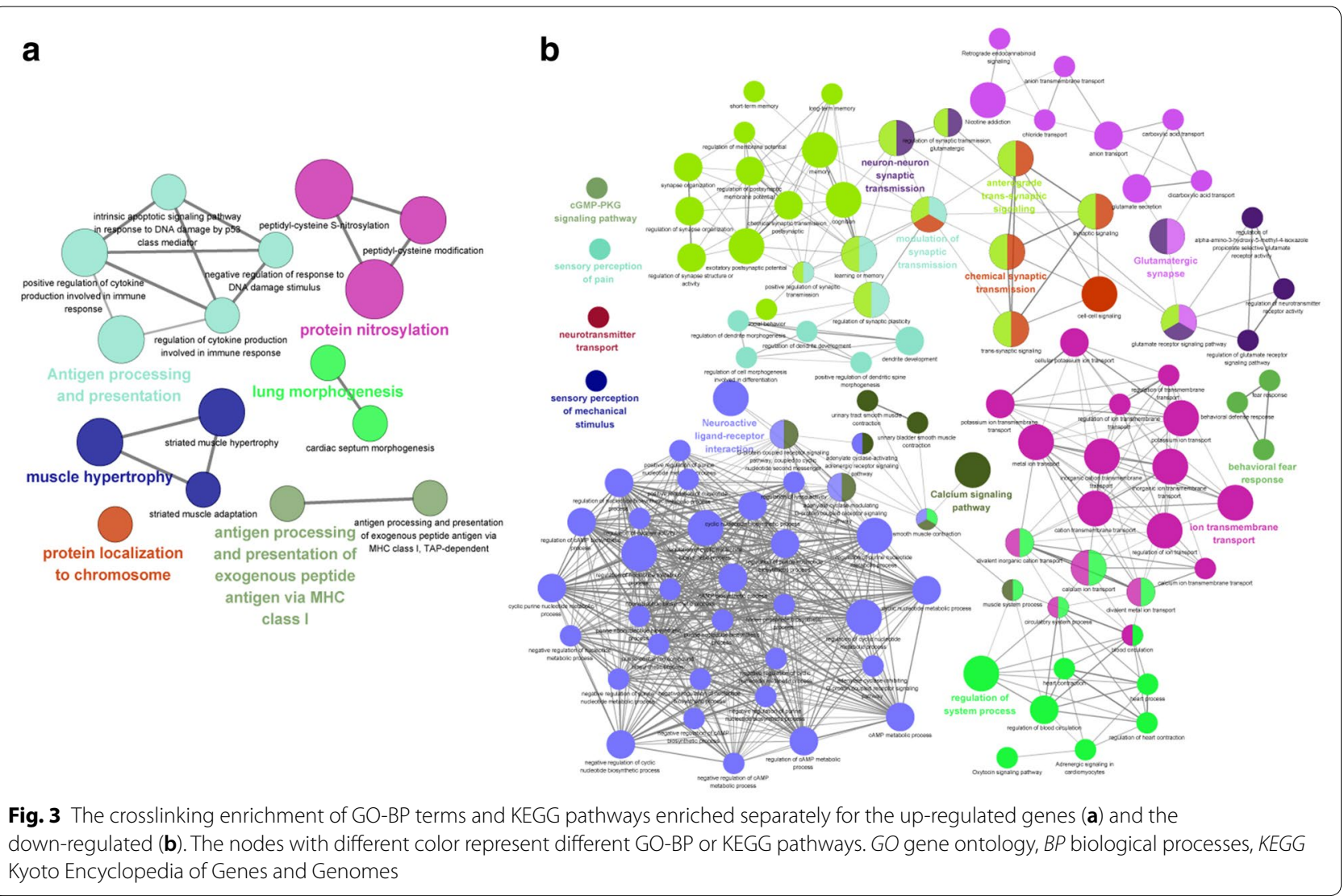

GRM1, GRM2 and GRM7 had higher degrees in module 1 , which were enriched with neuroactive ligandreceptor interaction, calcium signaling pathway and glutamatergic synapse in KEGG pathways in this study. Chen et al. [34] demonstrated that neuroactive ligandreceptor interactions are mainly associated with DIPG. Calcium is an essential signal transduction element that regulate numerous eukaryotic cellular functions including cell cycle progression [35]. Deregulation of the calcium signalling is linked to each of the 'cancer hallmarks' [36]. Additionally, the expression of glutamatergic system is implicated in tumour biology [37]. Given the role of these pathways in cancers, we speculated that GRM1, GRM2 and GRM7 may be associated with DIPG progression by involving in these pathways.

GRM1, GRM2 and GRM7 belong to the glutamate receptor family, which included ionotropic glutamate receptors (iGluR) and metabotropic glutamate receptors (mGluR) [38]. The mGluRs are further divided into three groups, among which, GRM1 is owned by group I, while GRM2 and GRM7 belong to group II and III respectively [39]. Emerging evidence has suggested a role for glutamate and its receptors in the biology of cancer. Glutamate receptor antagonists could limit tumor growth [40].
Blocking expression of selected GluR subunits inhibits proliferation of cancer cells in vitro [41]. Importantly, it has been demonstrated that glutamate receptor subunits are expressed in a variety of tumors, including glioma [42]. Previous studies reported that the aberrant expression of GRM1 induced spontaneous melanoma development in vivo [43, 44]. Brocke et al. [45] demonstrated that tumor growth may be suppressed via interfering with glutamate signaling, and suggested that glutamate receptor modulators may be an adjunctive treatment for central nervous system tumors. The study has shown that the GRM2 had huge potential for treating psychiatric and neurological diseases throughout the mammalian central nervous system, and that have been proposed as major targets for the development of drugs for human psychiatric and neurological diseases [46]. Recently, Ma et al. [47] reported that GRM2 was downregulated in glioma cells, and was regulated by eight transcription factors. In our study, GRM2 had higher degree in the PPI network. Previous study has reported that hubs that are "highly connected" in a PPI network tend to correspond to essential genes, which is called the "centrality-lethality rule" [48]. All these results may suggest the essential role of GRM2 in glioma. Furthermore, De et al. [49] revealed 


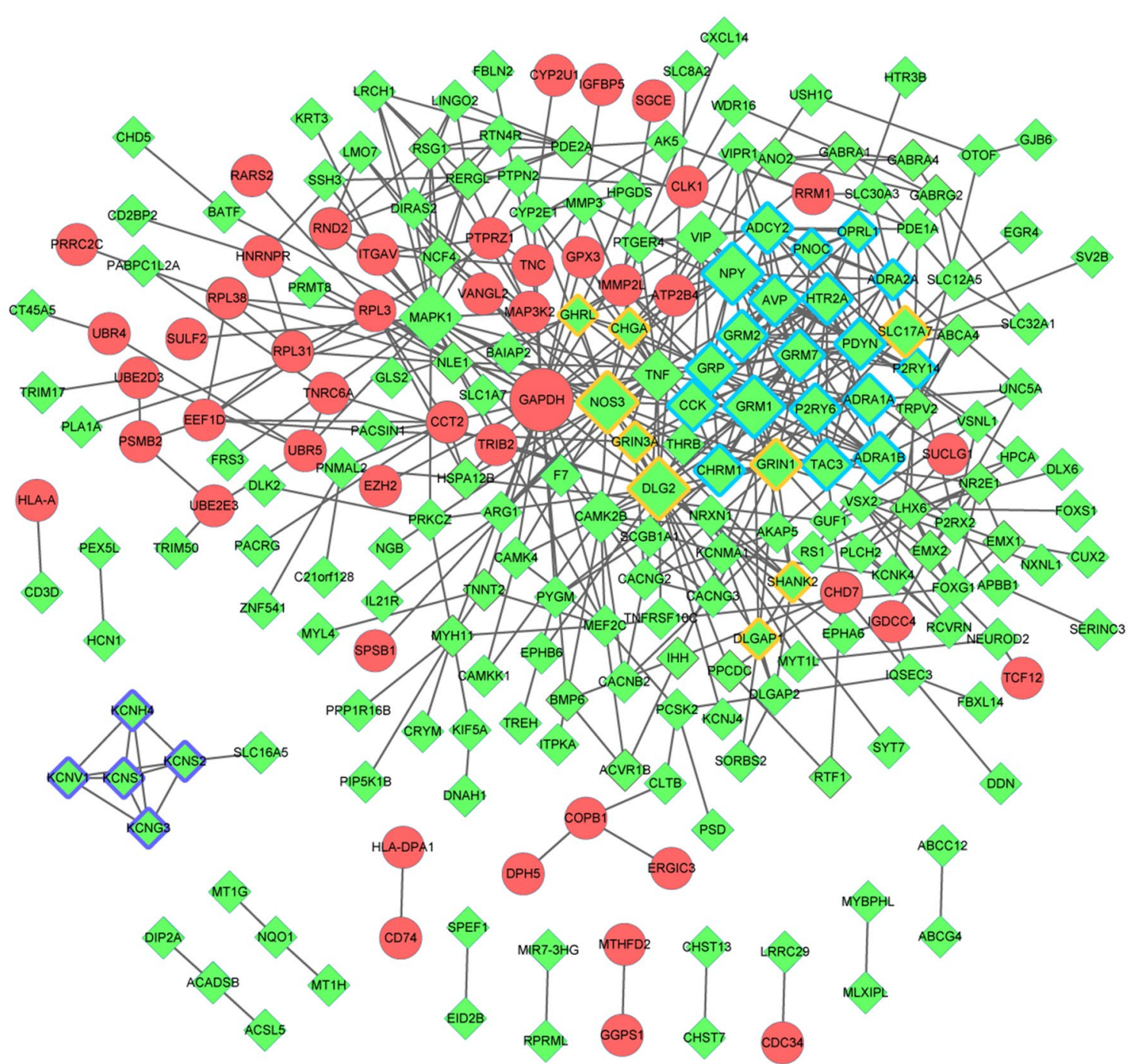

Fig. 4 Protein-protein interaction network constructed for the DEGs. The red circle and the green rhombus represent up-regulated genes and down-regulated genes, respectively. The nodes in Module 1, Module 2 and Module 3 separately were marked by blue, purple and yellow edges. DEGs differentially-expressed genes

Table 2 The differentially-expressed genes (DEGs) with a degree $>10$ in the protein-protein interaction (PPI) network

\begin{tabular}{lllllllll}
\hline Gene & Description & Degree & Gene & Description & Degree & Gene & Description & Degree \\
\hline GAPDH & Up & 27 & CAMK2B & Down & 16 & HTR2A & Down & 12 \\
MAPK1 & Down & 23 & AVP & Down & 16 & TAC3 & Down & 12 \\
NPY & Down & 22 & TNF & Down & 16 & VIP & Down & 11 \\
DLG2 & Down & 22 & CCK & Down & 15 & ADRA1A & Down & 11 \\
NOS3 & Down & 20 & GRIN1 & Down & 15 & CHRM1 & Down & 11 \\
GRM1 & Down & 20 & GRM7 & Down & 15 & ADRA1B & Down & 10 \\
GRP & Down & 16 & ADCY2 & Down & 14 & P2RY6 & Down & 10 \\
PDYN & Down & 16 & GRM2 & Down & 14 & SLC17A7 & Down & 10 \\
\hline
\end{tabular}


Table 3 The enriched pathways for the nodes in module 1 and 3

\begin{tabular}{|c|c|c|c|}
\hline Pathway name & Count & $p$-value & Genes \\
\hline \multicolumn{4}{|l|}{ Module 1} \\
\hline hsa04080:neuroactive ligand-receptor interaction & 11 & $7.94 \mathrm{E}-12$ & $\begin{array}{l}\text { P2RYG, GRM2, OPRL1, P2RY14, CHRM1, GRM7, } \\
\text { ADRA2A, ADRA1B, ADRA1A, GRM1, HTR2A }\end{array}$ \\
\hline hsa04020:calcium signaling pathway & 6 & $1.83 \mathrm{E}-05$ & ADCY2, CHRM1, ADRA1B, ADRA1A, GRM1, HTR2A \\
\hline hsa04724:glutamatergic synapse & 4 & $1.39 \mathrm{E}-03$ & $A D C Y 2, G R M 2, G R M 7, G R M 1$ \\
\hline hsa04022:CGMP-PKG signaling pathway & 4 & $4.08 \mathrm{E}-03$ & $A D C Y 2, A D R A 2 A, A D R A 1 B, A D R A 1 A$ \\
\hline hsa04970:salivary secretion & 3 & $1.26 \mathrm{E}-02$ & $A D C Y 2, A D R A 1 B, A D R A 1 A$ \\
\hline hsa04540:gap junction & 3 & $1.32 \mathrm{E}-02$ & ADCY2, GRM1, HTR2A \\
\hline hsa04270:vascular smooth muscle contraction & 3 & $2.34 \mathrm{E}-02$ & $A D C Y 2, A D R A 1 B, A D R A 1 A$ \\
\hline hsa04261:adrenergic signaling in cardiomyocytes & 3 & $3.42 \mathrm{E}-02$ & $A D C Y 2, A D R A 1 B, A D R A 1 A$ \\
\hline \multicolumn{4}{|l|}{ Module 3} \\
\hline hsa04724:glutamatergic synapse & 5 & $2.37 E-06$ & SLC17A7, DLGAP1, GRIN1, GRIN3A, SHANK2 \\
\hline hsa05033:nicotine addiction & 3 & $6.74 \mathrm{E}-04$ & SLCITA7, GRIN1, GRIN3A \\
\hline hsa04024:CAMP signaling pathway & 3 & $1.56 \mathrm{E}-02$ & GRIN1, GHRL, GRIN3A \\
\hline hsa05030:cocaine addiction & 2 & $4.86 \mathrm{E}-02$ & GRIN1, GRIN3A \\
\hline
\end{tabular}

that GRM7 was associated with mood disorders, suicide, and treatment response. GRM7 has been reported to be hypermethylated in breast cancer cells [50]. Specially, the expression level of GRM7 was validated in human glioma cell line U251. Taken together, these suggested that the GRM1, GRM2 and GRM7 might function in DIPG.

In this study, HTR $2 A$ had a higher degree and was strongly related with neuroactive ligand-receptor interactions. Besides, it was demonstrated to be down-regulated in U251 cells. Li et al. [51] have demonstrated that $H T R 2 A$ are related with low-grade and high-grade gliomas via neuroactive ligand-receptor interactions. Thus, the present results suggested that $H T R 2 A$ might play an important role in gliomas via neuroactive ligand-receptor interactions, which was consistent with previous findings. Besides, HTR2A was also enriched in calcium signaling pathway, meanwhile, $A D C Y 2$ was significantly related with the calcium signaling pathway as well. Being consistent with these results, Deng et al. [10] demonstrate that $A D C Y 2$ plays a role by the calcium signaling pathway in DIPG tumorigenesis. Hall et al. [52] showed that NPY-immunopositive played an important role in modulating cortical excitability of interneurons. NPY had a higher degree in the PPI network, suggesting that NPY might function in CNS.

It has been well known that TFs promote or repress transcription at a pre-transcription stage [26], while miRNA plays an important regulatory roles at the posttranscriptional level [25]. Many previous studies have investigated the pathogenesis of DIPG from transcriptional level using glioma cell line U251 [53-55]. In the present study, unlike the other studies using GSE50021 for analysis, we performed miRNA-TF-target gene regulatory network analysis in order to predict the TFs and miRNAs that may play roles in DIPG. TFAP2C was identified as candidate TF, which was a significantly down-regulated functional gene. However, result of PCR analysis showed that TFAP2C was upregulated in human glioma cell line U251, which was inconsistent with the prediction result. The contradiction may be due to the heterogeneity between tissue samples and cell samples. Further experiment is needed to detect the expression level of TFAP2C in DIPG. Study has suggested that TFAP2C promotes lung tumorigenesis by up-regulation of TGFBR1 and consequent activation of PAK1 signaling [56]. Another study showed that through regulation of RET, the expression of TFAP2C decreased in luminal breast cancer. Besides, EGFR and HER2 were regulated by TFAP2C in breast cancer [57]. Therefore, $T F A P 2 C$ may play a role in cancer. The $h s a-m i R-26 b-5 p$ had a higher degree in the TF-miRNA-target regulatory network, which may be related to DIPG. The research indicated that proliferation and apoptosis in lung cancer cells were inhibited via a $m i R-26 b-5 p$-EZH2-mediated approach [58]. Although the roles of TFAP2C and $h s a-m i R-26 b-5 p$ in DIPG have not been discussed, we inferred that TFAP2C and $h s a-m i R-26 b-5 p$ may play roles in DIPG tumorigenesis. Further genetic studies are required to verify this hypothesis.

Specially, the present study used real-time PCR to verify the expression of key genes GRM2, GRM7, HTR2A and TFAP2C through human glioma cell line U251. GRM7 and HTR2A were significantly downregulated in U251 cells compared with that in HEB cells, which was in accordance with the analysis results. GRM2 was not detected in cells, which may be due to its expression level 


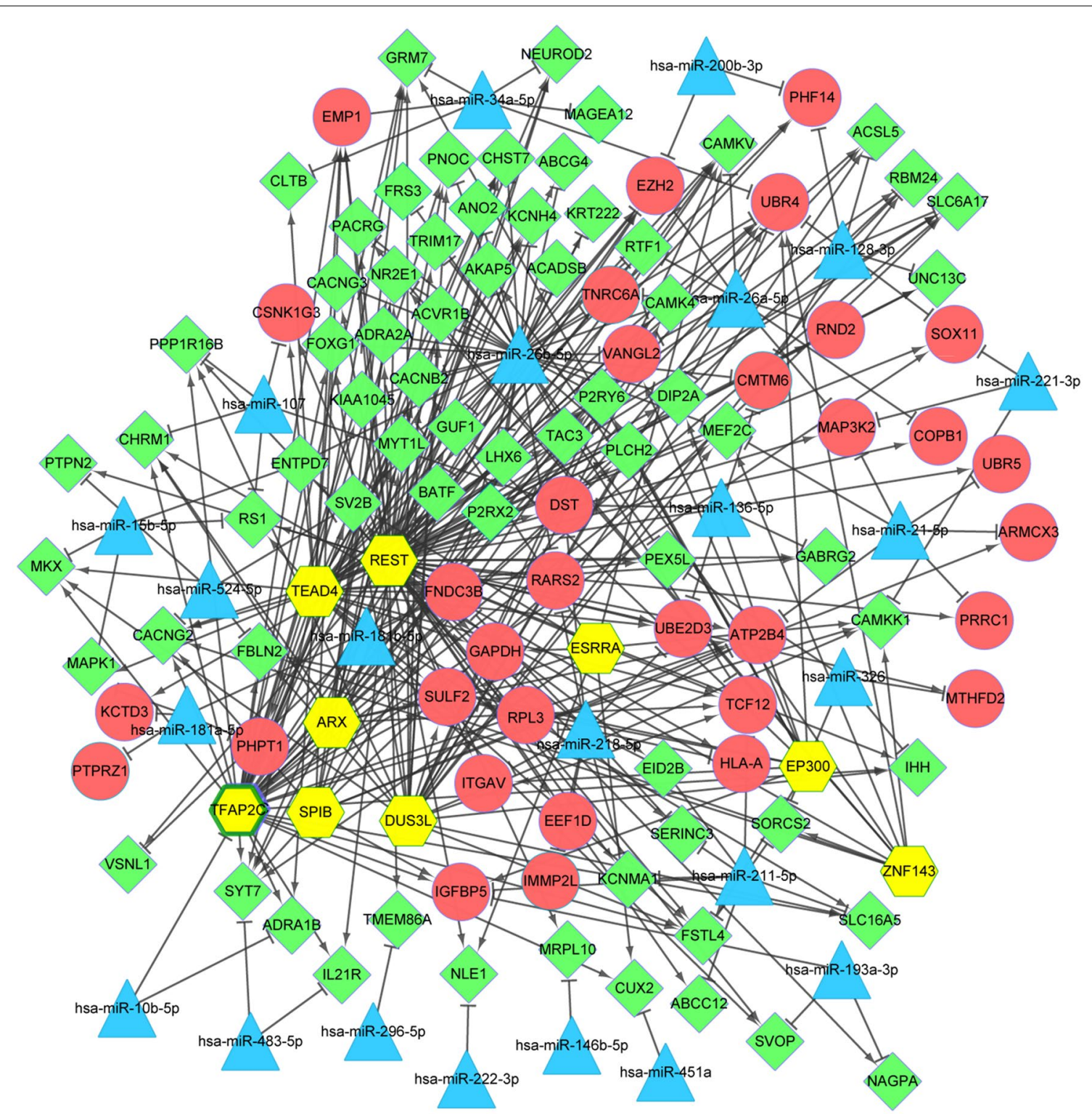

Fig. 5 The miRNA-TF-target regulatory network. The red circle and the green rhombus represent up-regulated genes and down-regulated genes, respectively. The blue triangle represents miRNA, and yellow hexagon indicates TFs. T shape represents the miRNA-target relationship, and arrow represents TF-target relationship. DEGs differentially-expressed genes, TFs transcription factors, miRNAs microRNAs

Table 4 The differentially-expressed genes (DEGs) with a degree $>10$ in the transcription factors-microRNAs-target regulatory network

\begin{tabular}{llllll}
\hline Gene & Description & Degree & Gene & Description & Degree \\
\hline REST & TF & 71 & hsa-miR-218-5p & miRNA & 15 \\
hsa-miR-26b-5p & miRNA & 41 & SPIB & TF & 12 \\
TFAP2C & TF/down & 39 & EP300 & TF & 12 \\
TEAD4 & TF & 36 & hsa-miR-26a-5p & miRNA & 11 \\
DUS3L & TF & 24 & ZNF143 & TF & 10 \\
ARX & TF & 15 & UBR4 & Up & 10 \\
\hline
\end{tabular}



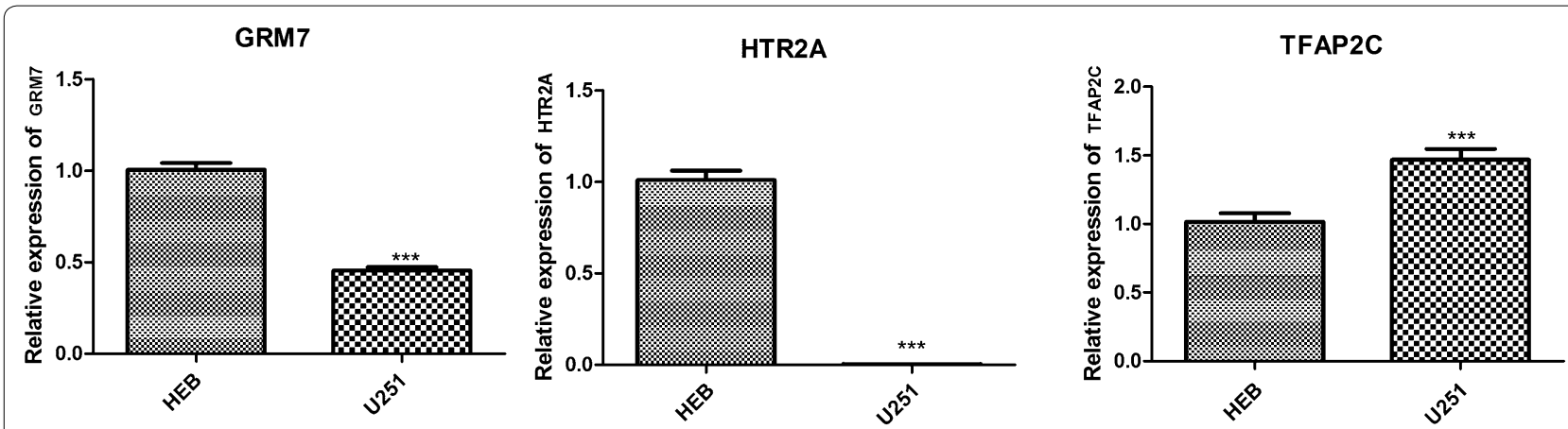

Fig. 6 The gene expression levels of GRM7, HTR2A and TFAP2C detected by real-time polymerase chain reaction. ${ }^{* * *} p<0.001$ compared with control

being too low in U251 cells or the difference between tissue and cell samples. TFAP2C was upregulated in human glioma cell line U251, being inconsistent with the prediction result, which needed to be further investigated. In the study of Buczkowicz et al. [2], the data was obtained from tumor tissue biopsy vs. normal. It is possible that different cell types contribute to gene expression change, therefore it is really a limitation that only one cell line was used for gene validation. We will continue collecting tissue samples for validation in the future.

In conclusion, this study indicated that GRM1, GRM7 and HTR2A might function in DIPG through the calcium signaling pathway and the neuroactive ligand-receptor interaction pathway. Meanwhile, TFAP2C and hsa-miR$26 b-5 p$ might have critical roles in the tumorigenesis of DIPG. This study provides new insights into the molecular mechanisms for the progress of DIPG and suggests directions for future study.

\section{Highlights}

1. A total of 378 differentially-expressed genes were identified.

2. We found 490 protein-protein interactions, 9 transcription factors and 27 microRNAs.

3. GRM1, HTR2A, TFAP2C and hsa-miR-26b-5p might be related to diffuse intrinsic pontine glioma.

\section{Abbreviations}

DIPG: diffuse intrinsic pontine glioma; DEGs: differentially-expressed genes; PPI: protein-protein interaction; TF: transcription factor; RAM: robust multiarray average; BP: biological process; MF: molecular function; CC: cellular component.

\section{Authors' contributions}

FH carried out the conception and design of the research, participated in the acquisition of data and drafted the manuscript. WZ carried out the analysis and interpretation of data. BY participated in the Statistical analysis. WC participated in the design of the study and performed the statistical analysis. LW conceived of the study, and participated in its design and coordination and helped to draft the manuscript. All authors read and approved the final manuscript.

\section{Author details}

${ }^{1}$ Department of Rehabilitation, Shanghai General Hospital, Shanghai Jiao Tong University, No. 100, Haining Road, Shanghai 200080, China. ${ }^{2}$ School of International Medical Technology, Shanghai Sanda University, No. 2727, Jinhai Road, Shanghai 201209, China.

\section{Acknowledgements}

Not applicable.

\section{Competing interests}

The authors declare that they have no competing interests.

\section{Availability of data and materials}

Not applicable. This study was only the primary research, and further study has been in progress.

\section{Consent for publication \\ Not applicable.}

\section{Ethics approval and consent to participate}

This study was approved by Ethics Committee of Shanghai Jiao Tong University and Shanghai Sanda University.

\section{Funding}

None.

\section{Publisher's Note}

Springer Nature remains neutral with regard to jurisdictional claims in published maps and institutional affiliations.

Received: 18 December 2017 Accepted: 12 August 2018

Published online: 20 August 2018

\section{References}

1. Vanan MI, Eisenstat DD. DIPG in children - what can we learn from the past? Front Oncol. 2015;5:237.

2. Buczkowicz P, Hoeman C, Rakopoulos P, Pajovic S, Letourneau L, Dzamba M, Morrison A, Lewis P, Bouffet E, Bartels U, Zuccaro J, Agnihotri S, Ryall S, Barszczyk M, Chornenkyy Y, Bourgey M, Bourque G, Montpetit A, Cordero F, Castelo-Branco P, Mangerel J, Tabori U, Ho KC, Huang A, Taylor KR, Mackay A, Bendel AE, Nazarian J, Fangusaro JR, Karajannis MA, Zagzag D, Foreman NK, Donson A, Hegert JV, Smith A, Chan J, Lafay-Cousin L, Dunn S, Hukin J, Dunham C, Scheinemann K, Michaud J, Zelcer S, Ramsay D, Cain J, Brennan C, Souweidane MM, Jones C, Allis CD, Brudno M, Becher $\mathrm{O}$, Hawkins $\mathrm{C}$. Genomic analysis of diffuse intrinsic pontine gliomas identifies three molecular subgroups and recurrent activating ACVR1 mutations. Nat Genet. 2014;46(5):451-6. 
3. Donaldson SS, Laningham F, Fisher PG. Advances toward an understanding of brainstem gliomas. J Clin Oncol. 2006;24(8):1266-72.

4. Hargrave D, Bartels U, Bouffet E. Diffuse brainstem glioma in children: critical review of clinical trials. Lancet Oncol. 2006;7(3):241-8.

5. Grasso CS, Tang Y, Truffaux N, Berlow NE, Liu L, Debily MA, Quist MJ, Davis LE, Huang EC, Woo PJ, Ponnuswami A, Chen S, Johung TB, Sun W, Kogiso M, Du Y, Qi L, Huang Y, Hutt-Cabezas M, Warren KE, Le Dret L, Meltzer PS, Mao H, Quezado M, van Vuurden DG, Abraham J, Fouladi M, Svalina MN, Wang N, Hawkins C, Nazarian J, Alonso MM, Raabe EH, Hulleman E, Spellman PT, Li XN, Keller C, Pal R, Grill J, Monje M. Functionally defined therapeutic targets in diffuse intrinsic pontine glioma. Nat Med. 2015;21(6):555-9.

6. Monje M, Mitra SS, Freret ME, Raveh TB, Kim J, Masek M, Attema JL, Li G, Haddix T, Edwards MS, Fisher PG, Weissman IL, Rowitch DH, Vogel $H$, Wong AJ, Beachy PA. Hedgehog-responsive candidate cell of origin for diffuse intrinsic pontine glioma. Proc Natl Acad Sci USA. 2011;108(11):4453-8.

7. Hashizume R, Smirnov I, Liu S, Phillips JJ, Hyer J, McKnight TR, Wendland M, Prados M, Banerjee A, Nicolaides T, Mueller S, James CD, Gupta N. Characterization of a diffuse intrinsic pontine glioma cell line: implications for future investigations and treatment. J Neurooncol. 2012;110(3):305-13.

8. Nakada M, Niska JA, Tran NL, McDonough WS, Berens ME. EphB2/R-Ras signaling regulates glioma cell adhesion, growth, and invasion. Am J Pathol. 2005;167(2):565-76.

9. Holland EC, Celestino J, Dai C, Schaefer L, Sawaya RE, Fuller GN. Combined activation of Ras and Akt in neural progenitors induces glioblastoma formation in mice. Nat Genet. 2000;25(1):55-7.

10. Deng L, Xiong P, Luo Y, Bu X, Qian S, Zhong W. Bioinformatics analysis of the molecular mechanism of diffuse intrinsic pontine glioma. Oncol Lett. 2016;12(4):2524-30.

11. Xi Y, Tang W, Yang S, Li M, He Y, Fu X. Mining the glioma susceptibility genes in children from gene expression profiles and a methylation database. Oncol Lett. 2017;14(3):3473-9.

12. Barrett T, Suzek TO, Troup DB, Wilhite SE, Ngau W-C, Ledoux P, Rudnev D, Lash AE, Fujibuchi W, Edgar R. NCBI GEO: mining millions of expression profiles_database and tools. Nucleic Acids Res. 2005;33(suppl 1):D562-6.

13. Gautier L, Cope L, Bolstad BM, Irizarry RA. affy —analysis of Affymetrix GeneChip data at the probe level. Bioinformatics. 2004;20(3):307-15.

14. Smyth GK. Limma: linear models for microarray data. In: Gentleman R, Carey VJ, Huber W, Irizarry RA, Dudoit S, editors. Bioinformatics and computational biology solutions using R and bioconductor. New York: Springer; 2005. p. 397-420.

15. Ashburner M, Ball CA, Blake JA, Botstein D, Butler H, Cherry JM, Davis AP, Dolinski K, Dwight SS, Eppig JT, Harris MA, Hill DP, Issel-Tarver L, Kasarskis A, Lewis S, Matese JC, Richardson JE, Ringwald M, Rubin GM, Sherlock G. Gene Ontology: tool for the unification of biology. Nat Genet. 2000;25(1):25-9.

16. Kanehisa M, Goto S. KEGG: Kyoto Encyclopedia of Genes and Genomes. Nucleic Acids Res. 2000;28(1):27-30.

17. Du J, Yuan Z, Ma Z, Song J, Xie X, Chen Y. KEGG-PATH: Kyoto Encyclopedia of Genes and Genomes-based pathway analysis using a path analysis model. Mol BioSyst. 2014;10(9):2441-7.

18. Huang DW, Sherman BT, Lempicki RA. Systematic and integrative analysis of large gene lists using DAVID bioinformatics resources. Nat Protoc. 2008:4(1):44-57.

19. Bindea G, Galon J, Mlecnik B. CluePedia Cytoscape plugin: pathway insights using integrated experimental and in silico data. Bioinformatics. 2013;29:661-3.

20. Bindea G, Mlecnik B, Hackl H, Charoentong P, Tosolini M, Kirilovsky A, Fridman W-H, Pagès F, Trajanoski Z, Galon J. ClueGO: a Cytoscape plug-into decipher functionally grouped gene ontology and pathway annotation networks. Bioinformatics. 2009;25(8):1091-3.

21. Shannon P, Markiel A, Ozier O, Baliga NS, Wang JT, Ramage D, Amin N, Schwikowski B, Ideker T. Cytoscape: a software environment for integrated models of biomolecular interaction networks. Genome Res. 2003;13(11):2498-504.

22. Szklarczyk D, Franceschini A, Wyder S, Forslund K, Heller D, Huerta-Cepas J, Simonovic M, Roth A, Santos A, Tsafou KP. STRING v10: protein-protein interaction networks, integrated over the tree of life. Nucleic Acids Res. 2014;43:D447-52.
23. Tang Y, Li M, Wang J, Pan Y, Wu F-X. CytoNCA: a Cytoscape plugin for centrality analysis and evaluation of protein interaction networks. BioSystems. 2015;127(Complete):67-72.

24. Bandettini WP, Kellman P, Mancini C, Booker OJ, Vasu S, Leung SW, Wilson JR, Shanbhag SM, Chen MY, Arai AE. MultiContrast Delayed Enhancement (MCODE) improves detection of subendocardial myocardial infarction by late gadolinium enhancement cardiovascular magnetic resonance: a clinical validation study. J Cardiovasc Magn Reson. 2012;14:83.

25. Slezakprochazka I, Durmus S, Kroesen BJ, Van den Berg A. MicroRNAs, macrocontrol: regulation of miRNA processing. RNA. 2010;16(6):1087-95.

26. Zaret KS, Carroll JS. Pioneer transcription factors: establishing competence for gene expression. Genes Dev. 2011;25(21):2227-41.

27. Delfino KR, Rodriguez-Zas SL. Transcription factor-microRNA-target gene networks associated with ovarian cancer survival and recurrence. PLoS ONE. 2013;8(3):12.

28. Dweep H, Gretz N. miRWalk2.0: a comprehensive atlas of microRNAtarget interactions. Nat Methods. 2015;12(8):697.

29. RS Janky, Verfaillie A, Imrichová H, Van de Sande B, Standaert L, Christiaens V, Hulselmans G, Herten K, Naval Sanchez M, Potier D, Svetlichnyy D, Kalender Atak Z, Fiers M, Marine J-C, Aerts S. iRegulon: from a gene list to a gene regulatory network using large motif and track collections. PLoS Comput Biol. 2014;10(7):e1003731.

30. Matys V, Fricke E, Geffers R, Gößling E, Haubrock M, Hehl R, Hornischer K, Karas D, Kel AE, Kel-Margoulis OV. TRANSFAC ${ }^{\circledR}$ : transcriptional regulation, from patterns to profiles. Nucleic Acids Res. 2003;31(1):374-8.

31. Matys V, Kelmargoulis OV, Fricke E, Liebich I, Land S, Barredirrie A, Reuter I, Chekmenev D, Krull M, Hornischer K. TRANSFAC and its module TRANSCompel: transcriptional gene regulation in eukaryotes. Nucleic Acids Res. 2006;34(suppl_1):D108-10.

32. Sloan CA, Chan ET, Davidson JM, Malladi VS, Strattan JS, Hitz BC, Gabdank 1. Narayanan AK, Ho M, Lee BT. ENCODE data at the ENCODE portal. Nucleic Acids Res. 2016;44(d1):D726-32.

33. Xu D, Ma P, Gao G, Gui Y, Niu X, Jin B. MicroRNA-383 expression regulates proliferation, migration, invasion, and apoptosis in human glioma cells. Tumor Biol. 2015;36(10):7743-53.

34. Chan KM, Fang D, Gan H, Hashizume R, Yu C, Schroeder M, Gupta N, Mueller S, James CD, Jenkins R, Sarkaria J, Zhang Z. The histone H3.3K27M mutation in pediatric glioma reprograms H3K27 methylation and gene expression. Genes Dev. 2013;27(9):985-90.

35. Mariot P, Vanoverberghe K, Lalevee N, Rossier MF, Prevarskaya N. Overexpression of an alpha $1 \mathrm{H}$ (Cav3.2) T-type calcium channel during neuroendocrine differentiation of human prostate cancer cells. J Biol Chem. 2002;277(13):10824-33.

36. Stewart TA, Yapa KT, Monteith GR. Altered calcium signaling in cancer cells. Biochim Biophys Acta. 2015;1848(10 Pt B):2502.

37. Kalariti N, Pissimissis N, Koutsilieris M. The glutamatergic system outside the CNS and in cancer biology. Expert Opin Investig Drugs. 2005;14(12):1487-96.

38. Conn PJ, Pin JP. Pharmacology and functions of metabotropic glutamate receptors. Annu Rev Pharmacol Toxicol. 1997;37:205-37.

39. Frati C, Marchese C, Fisichella G, Copani A, Nasca MR, Storto M, Nicoletti F. Expression of functional mGlu5 metabotropic glutamate receptors in human melanocytes. J Cell Physiol. 2000;183(3):364-72.

40. Nicoletti F, Arcella A, lacovelli L, Battaglia G, Giangaspero F, Melchiorri D. Metabotropic glutamate receptors: new targets for the control of tumor growth? Trends Pharmacol Sci. 2007;28(5):206-13.

41. Watanabe K, Kanno T, Oshima T, Miwa H, Tashiro C, Nishizaki T. The NMDA receptor NR2A subunit regulates proliferation of MKN45 human gastric cancer cells. Biochem Biophys Res Commun. 2008;367(2):487-90.

42. de Groot JF, Piao Y, Lu L, Fuller GN, Yung WK. Knockdown of GluR1 expression by RNA interference inhibits glioma proliferation. J Neurooncol. 2008;88(2):121-33.

43. Zhu H, Reuhl K, Botha R, Ryan K, Wei J, Chen S. Development of early melanocytic lesions in transgenic mice predisposed to melanoma. Pigment Cell Res. 2000;13(3):158-64.

44. Zhu H, Reuhl K, Zhang X, Botha R, Ryan K, Wei J, Chen S. Development of heritable melanoma in transgenic mice. J Invest Dermatol. 1998;110(3):247-52

45. Brocke KS, Staufner C, Luksch H, Geiger KD, Stepulak A, Marzahn J, Schackert G, Temme A, Ikonomidou C. Glutamate receptors in pediatric tumors of the central nervous system. Cancer Biol Ther. 2010;9(6):455-68. 
46. Li ML, Hu XQ, Li F, Gao WJ. Perspectives on the mGluR2/3 agonists as a therapeutic target for schizophrenia: still promising or a dead end? Prog Neuropsychopharmacol Biol Psychiatry. 2015;60:66-76.

47. Ma K, Cheng Z, Sun L, Li H. Identification of potential therapeutic targets for gliomas by bioinformatics analysis. Oncol Lett. 2017;14(5):5203-10.

48. Jeong H, Mason S, Barabasi A, Oltvai ZN. Lethality and centrality in protein networks. Nature. 2001:411(6833):41-2.

49. de Sousa RT, Loch AA, Carvalho AF, Brunoni AR, Haddad MR, Henter ID, Zarate CA, Machado-Vieira R. Genetic studies on the tripartite glutamate synapse in the pathophysiology and therapeutics of mood disorders. Neuropsychopharmacology. 2016;42:787.

50. Tryndyak $V$, Kovalchuk $O$, Pogribny IP. Identification of differentially methylated sites within unmethylated DNA domains in normal and cancer cells. Anal Biochem. 2006:356(2):202-7.

51. Li Y, Wang D, Wang L, Yu J, Du D, Chen Y, Gao P, Wang DM, Zhang F, Fu S. Distinct genomic aberrations between low-grade and high-grade gliomas of Chinese patients. PLOS ONE. 2013;8(2):22.

52. Hall S, Hunt M, Simon A, Cunnington LG, Carracedo LM, Schofield IS, Forsyth R, Traub RD, Whittington MA. Unbalanced peptidergic inhibition in superficial neocortex underlies spike and wave seizure activity. J Neurosci. 2015;35(25):9302-14.
53. Shi L, Zhang J, Pan T, Zhou J, Gong W, Liu N, Fu Z, You Y. MiR-125b is critical for the suppression of human U251 glioma stem cell proliferation. Brain Res. 2010;1312:120-6.

54. Liu C, Yao J, Mercola D, Adamson E. The transcription factor EGR-1 directly transactivates the fibronectin gene and enhances attachment of human glioblastoma cell line U251. J Biol Chem. 2000;275(27):20315-23.

55. Zhang Y, Chao T, Li R, Liu W, Chen Y, Yan X, Gong Y, Yin B, Qiang B. MicroRNA-128 inhibits glioma cells proliferation by targeting transcription factor E2F3a. J Mol Med. 2009;87(1):43.

56. Kim W, Kim E, Lee S, Kim D, Chun J, Park KH, Youn H, Youn B. TFAP2Cmediated upregulation of TGFBR1 promotes lung tumorigenesis and epithelial-mesenchymal transition. Exp Mol Med. 2016;48(11):e273.

57. De Andrade JP, Park JM, Gu VW, Woodfield GW, Kulak MV, Lorenzen AW, Wu VT, Van Dorin SE, Spanheimer PM, Weigel RJ. EGFR is regulated by TFAP2C in luminal breast cancer and is a target for vandetanib. Mol Cancer Ther. 2016;15:503-11.

58. Wu T, Chen W, Liu S, Lu H, Wang H, Kong D, Huang X, Kong Q, Ning Y, Lu Z. Huaier suppresses proliferation and induces apoptosis in human pulmonary cancer cells via upregulation of miR-26b-5p. FEBS Lett. 2014;588(12):2107-14.
Ready to submit your research? Choose BMC and benefit from:

- fast, convenient online submission

- thorough peer review by experienced researchers in your field

- rapid publication on acceptance

- support for research data, including large and complex data types

- gold Open Access which fosters wider collaboration and increased citations

- maximum visibility for your research: over 100M website views per year

At BMC, research is always in progress.

Learn more biomedcentral.com/submissions 